Revista Destaques Acadêmicos, Lajeado, v. 11, n. 3, 2019. ISSN 2176-3070

DOI: http://dx.doi.org/10.22410/issn.2176-3070.v11i3a2019.2331

http://www.univates.br/revistas

\title{
PREVALÊNCIA DE LESÕES EM ATLETAS DE TRIATHLON DE LONGA DISTÂNCIA
}

\author{
Anderson de Lazari ${ }^{1}$, Jéssica Luana Dornelles da Costa ${ }^{2}$, Leonardo Fin ${ }^{3}$, \\ Carlos Leandro Tiggeamnn ${ }^{4}$
}

Resumo: O triathlon é um esporte que une três distintas modalidades (natação, ciclismo e corrida) em provas que chegam a atingir centenas de quilômetros de percurso, exigindo altíssimo condicionamento e preparo físico dos atletas em vastos volume e quantidade de horas de treinamento. Em consequência, o risco de comprometer o corpo com a incidência de lesões ao sistema musculoesquelético é bastante recorrente. Diante disso, o presente estudo buscou avaliar a ocorrência de lesões esportivas provocadas pelos treinamentos físicos no triathlon através do histórico de lesões de 57 triatletas, distintos por sexo e idade, submetidos a entrevistas que questionavam seus perfis sociodemográficos, classificações de seus treinamentos e suas prevalências de lesões no último ano no esporte. A maioria dos participantes constituiu-se de homens com idade média de 34,5 anos, ensino superior completo e pertencentes às classes econômicas $\mathrm{A}, \mathrm{B}$ e C, com 1 a 3 anos de prática objetivando condicionamento e lazer, com frequências de treinos de 5 a 7 dias por semana. Do total, 73,7\% dos avaliados relataram ter sofrido lesão no último ano, sendo $92,9 \%$ ocorridas durante o treinamento de corrida, principalmente afetando locais distintos nos membros inferiores. Perante os resultados obtidos nesta pesquisa nos trazem um alto percentual de lesão encontrado entre os atletas avaliados. Nas três modalidades praticadas, a corrida se sobressai como a maior causadora de desgastes e ferimentos desencadeados, sobretudo, nos membros inferiores. Com isto, o acompanhamento profissional qualificado se mostra indispensável fazendo com que a carga nos treinos de corrida sejam moderadas dando uma maior importância a desconfortos e possíveis lesões nos membros inferiores.

Palavras-chave: Triathlon. Lesão. Natação. Ciclismo. Corrida.

1 1Graduado em Educação Física Bacharelado, Centro Universitário da Serra Gaúcha.

2 Graduada em Educação Física Bacharelado e Licenciatura, Universidade do Vale do TaquariUnivates, Profissional de Educação Física.

3 Graduando em Educação Física Bacharelado, Universidade do Vale do Taquari - Univates.

4 Doutor em Ciências do Movimento Humano, Universidade do Vale do Taquari - Univates, Docente. 


\section{INTRODUÇÃO}

O triathlon é um esporte que se define, basicamente, pela união de três modalidades, previamente estruturadas no que diz respeito à prática desportiva, que são, nesta ordem, a natação, o ciclismo e a corrida. A palavra grega Triathlon era utilizada para designar a prática de quaisquer três modalidades simultaneamente, porém nos anos 1970 a ideia por trás do termo retornou associando-se à prática consecutiva das atividades supracitadas (ANJOS; FERNANDES FILHO; NOVAES, 2003). Apesar de recente, se comparado a outros esportes, a inclusão como esporte olímpico no ano $2000 \mathrm{fez}$ o triathlon se popularizar por todo o mundo. No Brasil, a Confederação Brasileira de Triathlon (CBTri) é a entidade oficial para a organização do esporte e conta com 25 federações estaduais e mais de três mil atletas filiados (FERRACINI, 2015).

Caracterizado como uma prova de fundo, o triathlon assim como o ciclismo e as maratonas, é um desporto que interage diretamente com a resistência aeróbica de seus praticantes exigindo e promovendo elevadas adaptações em seus limiares anaeróbicos e no consumo máximo de oxigênio (PORTAL et al., 2004). Atualmente, o esporte divide-se em algumas categorias de acordo com a distância realizada nos três percursos, variando bastante as exigências nas provas, sendo elas e suas respectivas distâncias em natação, ciclismo e corrida: Sprint - 750 m, 20 km e 5 km; Olímpica - 1.5 km, 40 km e 10 km; Longa distância - 3 a 4,5 km, 80 a 120 km e 20 a 30 km; Mixed Relay - $300 \mathrm{~m}, 8 \mathrm{~km}$ e $2 \mathrm{~km}$; Ironman - $3.8 \mathrm{~km}, 180 \mathrm{~km}$ e $42 \mathrm{~km}$; e por último a MeioIronman com exatamente metade de cada das três distâncias (CBTRI, 2019).

Em geral, o treinamento de triathlon requer maior sacrifício em comparação aos outros esportes que compõem as etapas do triathlon. Para compensar as particularidades de cada prática, o triatleta tem treinamentos mais exaustivos e extenuantes, com aumento do volume e maior quantidade de horas por semana. Em consequência desta demasia o risco de comprometer o corpo é muito maior (FERRACINI, 2015). Já que o triathlon demanda de todas funções do sistema musculoesquelético, este pode desencadear contusões, fraturas e, com maior frequência, lesões musculares, tendinosas e ligamentares (EGERMANN et al., 2003; JUNIOR; MILARES, 2016; JACOMEL et al., 2008). Análises mostram que, além das lesões mais comuns encontradas em triatletas, fatores relacionados à prática ao ar livre influenciam no agravamento de traumatismos. O calor excessivo leva à desidratação e queimaduras solares, enquanto o frio demasiado é capaz de provocar hipotermia e broncoespasmo (BEZEM; BEZEM, 2009).

Através dos estudos de Cipriani, Swartz e Hudgson, (1998); Jacomel et al. (2008); Orgaratto e Toigo, (2010), foram avaliados 78 triatletas submetidos a entrevistas onde responderam questões sobre o número de lesões já sofridas em cada uma das três modalidades que compõe o esporte separadamente. $\mathrm{Na}$ natação constatou-se a predominância de bursites e tendinites, principalmente nos membros superiores, seguidas de casos bem menos prevalentes de 
dorsalgia e lombalgia. No ciclismo, as lesões epiteliais como cortes e contusões sobressaíram-se às lesões musculoesqueléticas e estas ocorrendo mais presentes na região de joelhos, tornozelos e coluna vertebral. Já na corrida, os joelhos e os membros inferiores como um todo são amplamente afetados em ordem decrescente nas áreas musculares, articulares, tendinosas, conjuntivas e ósseas, devido à solicitação e impacto durante a sustentação corporal e na força motriz ao longo das provas. Nas três modalidades, a maioria das lesões sucedeu-se durante os treinamentos e não nas competições.

Um estudo relata a prevalência de 130 lesões relacionadas ao triathlon, sendo $82 \%$ durante a fase final das provas, que é o momento da corrida. Nesta fase, o atleta está sobrecarregado da prova de ciclismo e a fadiga esforça exageradamente as estruturas corporais, principalmente atingindo os músculos lombares, isquiotibiais e o gastrocnemio (MCHARDY; POLLARD; FERNANDEZ, 2006). Também se verifica que atletas que já possuem histórico de esportes anteriores apresentam propensão às novas lesões e conjuntamente se correlaciona a incidência das lesões ao volume de treino (FERRACINI, 2015). Diante das prevalências de lesões provocadas pela prática do esporte, destaca-se a necessidade de estratégias de treinamento individual adequado às capacidades e dificuldades particulares de cada atleta, bem como a adequação dos instrumentos utilizados e o estudo da biomecânica envolvida nas fases de transição entre as etapas do triathlon (FERRACINI, 2015).

Para tanto, percebe-se ser essencial a compreensão dos mecanismos que acarretam em lesões para que se proceda às prevenções esportivas para a prática saudável do triathlon. A importância do trabalho se demonstra por todo o contexto citado anteriormente, levando-se em consideração fundamentalmente o impacto negativo que a lesão acarreta no cotidiano de um atleta no âmbito esportivo e social. A pesquisa se justifica também pela sua relevância acadêmica, podendo contribuir para o aprofundamento da temática no campo. Desta forma, o objetivo do presente estudo é avaliar a ocorrência de lesões esportivas provocadas pelos treinamentos físicos no triathlon através da constatação do histórico de lesões de triatletas amadores distintos por sexo e idade, detectando os tipos de lesões e as situações em que estas ocorreram.

\section{MATERIAIS E MÉTODOS}

O estudo caracteriza-se por uma pesquisa epidemiológica transversal descritiva. A amostra foi constituída por triatletas de ambos os sexos, com idade entre 18 a 50 anos, praticantes de triathlon de longa distância, selecionadas por conveniência em todo território nacional. Como critério de inclusão os participantes do estudo deveriam ser praticantes da modalidade por pelo menos seis meses. Os atletas poderiam ser tanto amadores como profissionais, mas que estivessem participando de provas de longa distância. Foram excluídos da pesquisa atletas que praticassem outra modalidade além de natação, ciclismo, 
corrida e musculação, também foram excluídos atletas com alguma deficiência física ou mental que pudessem interferir nas análises de desfecho.

Para desenvolvimento do estudo foi aplicado um questionário para avaliar o perfil dos atletas e também o nível de lesão em praticantes de triathlon de longa distância. Os participantes preencheram um questionário com 12 questões de perfil sociodemográfico e 23 questões sobre treinamento e lesões. O questionário foi construído a partir dos objetivos do estudo, apresentando questões abertas, fechadas e de múltipla escolha. Foi elaborado com questões específicas do perfil sociodemográfico (idade, profissão, estado, cidade, sexo, estado civil, graduação, renda, doenças e uso de medicamentos), sobre treinamento físico (tempo de prática, volume diário, volume semanal, tempo de duração e orientação) e sobre lesões (avaliação física, pontos anatômicos, tratamento, tempo de recuperação, esporte ocasionador da lesão e prevenção). Após a montagem da primeira versão do questionário, o mesmo foi entregue para um profissional de Educação Física, ex- atleta de triathlon com vasta experiência na área, para que o mesmo pudesse avaliar o conteúdo. Após seu retorno o questionário foi ajustado e considerado apto para avaliação dos objetivos propostos.

Tanto TCLE quanto o instrumento de coleta foram aplicados por meio de um formulário eletrônico (http://qualtrics.com), distribuído por correio eletrônico (e-mail), redes sociais dos pesquisadores (WhatsApp, Facebook e Instagram). Ao final das coletas, os dados foram exportados do sistema qualtrics.com para o software SPSS (Statistical Package for the Social Sciences, Nova York, Estados Unidos), versão 20, tendo os resultados expressos por média e desvio padrão, para dados contínuos e medidas relativas e absolutas para dados categóricos.

\section{RESULTADOS}

Inicialmente participaram do estudo 69 atletas de triathlon, destes, 12 foram excluídos por apresentarem respostas incompletas. Em relação ao perfil sociodemográfico apresentado na Tabela 1, a maioria dos atletas são do sexo masculino $(80,7 \%)$, com idade média de $34,5 \pm 8,2$ anos. Analisando os dados de estado civil, instituição e renda, a predominância está no público casado $(49,1 \%)$ e solteiro (31,6\%), ensino superior $(42,1 \%)$ e pós-graduado $(38,6 \%)$ e com a renda entre a classe A à C $(79 \%)$. Quanto a ingestão de bebida alcoólica e seus hábitos de tabagismo foi constatado que $(56,1 \%)$ ingerem bebidas alcoólicas e apenas $(5,3 \%)$ fumam. Em relação à prevalência de doenças crônicas, $(7 \%)$ apresentam e $(12,3 \%)$ fazem tratamento medicamentoso contínuo. 
Tabela 1: Perfil sociodemográfico $(n=57)$

\begin{tabular}{l|c|c}
\hline & $\mathbf{N = 5 7}$ & $\%$ \\
\hline Sexo (masculino) & 46 & 80,7 \\
\hline Estado civil & 18 & 31,6 \\
\hline Solteiro(a) & 8 & 14,0 \\
\hline União estável & 28 & 49,1 \\
\hline Casado(a) & 3 & 5,3 \\
\hline Divorciado(a) & 3 & \\
\hline Escolaridade & 24 & 5,3 \\
\hline Ensino médio & 22 & 42,1 \\
\hline Ensino superior & 8 & 38,6 \\
\hline Pós-graduação & & 14,0 \\
\hline Mestrado/Doutorado & 15 & 26,3 \\
\hline Classificação econômica & 12 & 21,1 \\
\hline Classe A & 18 & 31,6 \\
\hline Classe B & 8 & 14,0 \\
\hline Classe C & 4 & 7,0 \\
\hline Classe D & 3 & 5,3 \\
\hline Classe E & 32 & 56,1 \\
\hline Tabagismo & 4 & 7,0 \\
\hline Consumo de álcool & 1 & 25,0 \\
\hline Doenças crônicas & 2 & 50,0 \\
\hline Hipercolesterolemia & 1 & 25,0 \\
\hline Pressão alta & 7 & 12,3 \\
\hline Púrpura trombocitopenia & & \\
\hline Tratamento medicamentoso & & \\
\hline & & \\
\hline
\end{tabular}

Fonte: Elaborado pelo autor (2019)

Na Tabela 2 são apresentados os principais dados descritivos sobre a prática de treinamento de triathlon, sendo constatada a predominância de atletas que praticam a modalidade de 1 a 3 anos $(52,6 \%)$, quanto aos principais motivos para prática do triathlon foi a procura de lazer $(42,1 \%)$ e condicionamento físico $(40,4 \%)$. A frequência semanal de treinamento mais prevalente foi entre 5 á 7 dias por semana $(85,9 \%)$, com sessões diárias de 2 treinos por dia $(71,9 \%)$ e com tempo médio por sessão de treinamento entre 61 a 90 minutos $(57,9 \%)$. Na tabela temos também outras variáveis como volume de treinamento, modalidade com maior desgaste físico, orientação de treinamento, avaliações e atividades pré e pós treinamento. 
Tabela 2 - Classificações de treinamento e variáveis associadas $(n=57)$

\begin{tabular}{|c|c|c|}
\hline & $\mathbf{N}$ & $\%$ \\
\hline \multicolumn{3}{|l|}{ Tempo de triathlon } \\
\hline Até 1 ano & 4 & 7,0 \\
\hline de 1 a 3 anos & 30 & 52,6 \\
\hline de 4 a 5 anos & 14 & 24,6 \\
\hline de 6 a 10 anos & 5 & 8,8 \\
\hline Mais de 10 anos & 4 & 7,0 \\
\hline \multicolumn{3}{|c|}{ Motivos pela prática de triathlon * } \\
\hline Condicionamento físico & 23 & 40,4 \\
\hline Lazer & 24 & 42,1 \\
\hline Saúde & 16 & 28,1 \\
\hline Estético & 2 & 3,5 \\
\hline \multicolumn{3}{|l|}{ Categoria } \\
\hline Amador & 56 & 98,2 \\
\hline Profissional & 1 & 1,8 \\
\hline \multicolumn{3}{|l|}{ Treinamento semanal } \\
\hline 1 a 2 dias/semana & 2 & 3,5 \\
\hline 3 a 4 dias/semana & 6 & 10,5 \\
\hline 5 a 6 dias/semana & 21 & 36,8 \\
\hline 7 dias/semana & 28 & 49,1 \\
\hline \multicolumn{3}{|l|}{ Sessões diárias } \\
\hline 1 treino por dia & 13 & 22,8 \\
\hline 2 treinos por dia & 41 & 71,9 \\
\hline 3 treinos por dia & 3 & 5,3 \\
\hline \multicolumn{3}{|l|}{ Tempo médio por sessão } \\
\hline$\leq 30$ minutos por dia & 1 & 1,8 \\
\hline 31 a 60 minutos por dia & 9 & 15,8 \\
\hline 61 a 90 minutos por dia & 33 & 57,9 \\
\hline 91 a 120 minutos por dia & 12 & 21,1 \\
\hline$>120$ minutos por dia & 2 & 3,5 \\
\hline \multicolumn{3}{|l|}{ Volume semanal de natação } \\
\hline$\leq 6000 \mathrm{mts}$ & 18 & 31,6 \\
\hline 6001 a $8000 \mathrm{mts}$ & 15 & 26,3 \\
\hline 8001 a $10000 \mathrm{mts}$ & 13 & 22,8 \\
\hline 10001 a $12000 \mathrm{mts}$ & 7 & 12,3 \\
\hline$>12000 \mathrm{mts}$ & 4 & 7,0 \\
\hline
\end{tabular}




\begin{tabular}{|c|c|c|}
\hline & $\mathbf{N}$ & $\%$ \\
\hline \multicolumn{3}{|l|}{ Volume semanal de pedal } \\
\hline$\leq 100 \mathrm{~km}$ & 12 & 21,1 \\
\hline 101 a $200 \mathrm{~km}$ & 24 & 42,9 \\
\hline 201 a $300 \mathrm{~km}$ & 14 & 25,0 \\
\hline 301 a $400 \mathrm{~km}$ & 6 & 10,7 \\
\hline \multicolumn{3}{|l|}{ Volume semanal de pedal } \\
\hline$>400 \mathrm{~km}$ & 1 & 1,8 \\
\hline \multicolumn{3}{|l|}{ Volume semanal de corrida } \\
\hline$\leq 10 \mathrm{~km}$ & 1 & 1,8 \\
\hline 10,1 a $20 \mathrm{~km}$ & 5 & 8,8 \\
\hline 20,1 a $30 \mathrm{~km}$ & 15 & 26,3 \\
\hline 30,1 a $50 \mathrm{~km}$ & 25 & 43,9 \\
\hline$>50 \mathrm{~km}$ & 11 & 19,3 \\
\hline \multicolumn{3}{|l|}{ Desgastes físicos pelo esporte } \\
\hline Natação & 6 & 10,5 \\
\hline Ciclismo & 3 & 5,3 \\
\hline Corrida & 48 & 84,2 \\
\hline \multicolumn{3}{|l|}{ Orientação de treinamento personalizado* } \\
\hline Profissionais de Educação Física & 48 & 84,2 \\
\hline Outro profissional da saúde & 18 & 31,6 \\
\hline Atleta ou ex-atleta & 14 & 24,6 \\
\hline Planilha pronta da internet & 3 & 5,3 \\
\hline Utilização de aplicativos (Smartphone) & 9 & 15,8 \\
\hline \multicolumn{3}{|l|}{ Avaliações $\left(^{*}\right)$} \\
\hline Medidas corporais & 16 & 28,1 \\
\hline Testes de resistência & 7 & 12,3 \\
\hline Testes Força/agilidade/velocidade & 7 & 12,3 \\
\hline Avaliações realizadas por médicos & 25 & 43,9 \\
\hline \multicolumn{3}{|l|}{ Atividades pré e pós sessão de treinamento } \\
\hline Alongamento pré treinamento & 10 & 17,5 \\
\hline Aquecimento pré treinamento & 40 & 70,2 \\
\hline Alongamento pós treinamento & 26 & 45,6 \\
\hline
\end{tabular}

* questão com mais de uma possibilidade de resposta.

Fonte: Elaborado pelo autor (2019).

A Tabela 3 demonstra a prevalência de lesão dos atletas de triathlon de longa distância, momento da lesão, tratamento e tempo de recuperação. 
Tabela 3 - Prevalência de lesão dos atletas de triathlon de longa distância e variáveis associadas $(n=57)$

\begin{tabular}{|c|c|c|}
\hline & $\mathbf{N}$ & $\%$ \\
\hline Prevalência de lesão & 42 & 73,7 \\
\hline \multicolumn{3}{|l|}{ Local Muscular/Tendíneo * } \\
\hline Tendão do calcâneo & 14 & 33,3 \\
\hline Panturrilha & 11 & 26,2 \\
\hline Coxa Posterior & 7 & 16,7 \\
\hline Patela & 5 & 11,9 \\
\hline Manguito rotador & 4 & 9,5 \\
\hline Glúteo Máximo & 2 & 4,8 \\
\hline Grande Dorsal & 2 & 4,8 \\
\hline Abdominal/púbis & 1 & 2,4 \\
\hline Coxa Anterior & 0 & 0,0 \\
\hline Deltoide & 0 & 0,0 \\
\hline Outro & 7 & 16,7 \\
\hline \multicolumn{3}{|l|}{ Local Osteo/Articular * } \\
\hline Tornozelo/pé & 17 & 40,5 \\
\hline Tíbia & 6 & 14,3 \\
\hline Joelho & 5 & 11,9 \\
\hline Quadril & 3 & 7,1 \\
\hline Lombar & 3 & 7,1 \\
\hline Ombros & 3 & 7,1 \\
\hline Fêmur & 1 & 2,4 \\
\hline Coluna vertebral & 0 & 0,0 \\
\hline Cotovelos & 0 & 0,0 \\
\hline Punhos & 0 & 0,0 \\
\hline \multicolumn{3}{|l|}{ Momento da lesão * } \\
\hline Competição & 1 & 2,4 \\
\hline Treinamento de natação & 4 & 9,5 \\
\hline Treinamento de corrida & 39 & 92,9 \\
\hline Treinamento de bike & 2 & 4,8 \\
\hline Musculação & 2 & 4,8 \\
\hline \multicolumn{3}{|c|}{ Tratamento com profissional especializado* } \\
\hline Médico & 22 & 38,6 \\
\hline Fisioterapeuta & 35 & 61,4 \\
\hline Massagista & 8 & 14,0 \\
\hline
\end{tabular}




\begin{tabular}{l|c|c}
\hline & $\mathbf{N}$ & $\mathbf{\%}$ \\
\hline Tempo de recuperação & & \\
\hline 2 semanas & 7 & 17,1 \\
\hline 3 semanas & 11 & 26,8 \\
\hline 4 semanas & 6 & 14,6 \\
\hline$\geq 5$ semanas & 17 & 41,5 \\
\hline
\end{tabular}

* questão com mais de uma possibilidade de resposta.

Fonte: Elaborado pelo autor (2019).

\section{DISCUSSÃO}

De acordo com o objetivo do estudo que buscou avaliar a prevalência de lesões em praticantes de triathlon e as características envolvidas na ocorrência destas, encontrou-se alto percentual de casos entre os entrevistados. Diversos estudos apontam resultados muito semelhantes quanto ao número médio de prevalentes por ano no esporte (WILK; FISHER; RANGELLI, 1995; EGERMANN et al., 2003; PERRONI et al., 2006). Entre os relatos, apontase em totalidade o motivo da alta intensidade e carga horária de treinos vinculados ao desenvolvimento de três modalidades simultâneas. Dos momentos declarados de maior prevalência, o período de treinamento superou amplamente o de competição, pois o montante de tempo desempenhado em treino é demasiadamente superior, não havendo fatores adicionais a este que justifiquem tal resultado (EGERMANN et al., 2003). Já quanto à modalidade praticada, a alta proporção de lesões durante a corrida é igualmente referida por diversos autores que associam ao uso excessivo dos membros inferiores (região mais lesionada) descrevendo fatores como torções, escoriações e contusões promovidas durante as sucessões de impactos da ação (ANDERSEN et al., 2013; MIGLIORINI, 2011; VLECK et al., 2010).

Nos dados apresentados no perfil sociodemográfico, a grande maioria dos participantes, homens com idade média de 34,5 anos, ensino superior completo e pertencentes às classes econômicas $\mathrm{A}, \mathrm{B}$ e $\mathrm{C}$, assemelham-se ao padrão estatístico de praticantes da modalidade analisados em outros estudos nacionais (VECHIATO; COSTA, 2016; NAZARIO et al., 2011). Tal perfil é explicado pela grande exigência de tempo e dedicação necessária ao intenso regime de treinos, que restringe o acesso a pessoas com disponibilidade e boa estabilidade socioeconômica (COSCA; NAVAZIO, 2007).

Analisando itens mais vinculados às predições da saúde dos atletas, percebe-se um baixíssimo índice de tabagismo - condição bastante associada à redução de performance esportiva devido aos impactos que esta provoca no funcionamento do sistema respiratório (RABAHI, 2012). Já o consumo de álcool, registrado por pouco mais da metade dos entrevistados, principalmente quanto à saúde, é mau visto em alguns estudos e tido como indiferente por outros 
(MELONI; LARANJEIRA, 2004; GUIMARÃES, 2010). Sendo o seu uso muito associado à quantidade e frequência consumida, há estudos que mostram que o consumo moderado pode beneficiar o sistema cardiovascular e reduzir os riscos de doença arterial coronária e acidente vascular cerebral (BATLOUNI, 2006; WELLMANN et al., 2004; YUSUF et al., 2004). Dos quatro atletas que declararam possuir doenças crônicas, três descreveram ter disfunções cardiovasculares, taxa comumente baixa em atletas de esportes de resistência (ISHIDA, 2013). Um relatou ter doença rara e sem relação considerável ao esporte, assim como além destes quatro, outros três apontaram fazer tratamento medicamentoso contínuo sem relevância à prática de exercícios.

Em relação ao tempo de triathlon, fora uma pequena parcela que ainda não completou o primeiro ano, a maioria parte dos entrevistados praticam de um a três anos, seguido do grupo de praticantes de quatro a cinco anos e de poucos que se mantém do sexto ano em diante no esporte. Por ser um esporte de alto condicionamento físico, envolvendo forte comprometimento, poucos optam por dedicarem-se tantos anos e acabam por reduzir o ritmo em atividades de menor exigência (EPIPHANIO; AMATUZZI, 1997). Um estudo analisou o índice de lesão comparado ao tempo de prática e averiguou que entre os avaliados com apenas um ano foi registrado $14 \%$ de lesões, de dois a três anos $33 \%$, chegando a $45 \%$ nos com quatro anos ou mais no esporte, indicando outro motivo pela limitação da permanência (KORKIA; TUNSTALL-PEDOE DPHIL; MAFFULLI, 1994). Entre os motivos que levaram os participantes à prática, há quase em totalidade uma divisão relativamente semelhante entre a busca ao lazer, condicionamento e saúde, padrão motivacional relatado em artigos envolvendo triatletas amadores, categoria majoritária neste estudo (NAZARIO et al., 2011; WILK; FISHER; RANGELLI, 1995). Ainda alega-se que o prazer e o bem-estar são os objetivos mais buscados por amadores praticantes de esportes em geral (AGUIAR et al., 2010). A ínfima parcela declarada profissional ilustra a dificuldade requisitada para a prática de alto nível do triathlon, resultando no alto índice de amadores tanto nos voluntários deste estudo quanto em registros federativos (CBTRI, 2019).

Quanto à frequência dos treinos, o fato da grande maioria treinar de cinco a sete dias da semana e ainda duas vezes por dia, refletem a necessidade constante de estímulos para obterem-se os difíceis aprimoramentos do esporte e também de evitarem-se as reduções na resistência física e fisiológica do triatleta (RAMA, 2011). Há ainda um grande volume médio por sessão em que o maior número dos participantes se dedica de 61 a 90 minutos e uma parcela considerável ainda estende-se até 120 minutos. Tamanha frequência e volume são necessariamente demandados em virtude do objetivo principal do esporte, que é a execução de três provas cíclicas consideravelmente diferentes (ETXEBARRIA, 2019).

Todo esse montante de tempo dedicado ao triathlon gera um altíssimo volume semanal médio de exercícios em cada modalidade. A natação 
corresponde à primeira etapa de uma competição e a sua baixa proporção de distância percorrida por tempo, faz com que seu trajeto seja equivalente a somente $2,25 \%$ do percurso total em relação aos 78,68\% de ciclismo e $19,01 \%$ de corrida, em média nas distintas categorias do esporte (CBTri, 2019). Nos resultados dos volumes coletados neste estudo, proporcionalmente os avaliados praticam cerca de três vezes o ciclismo, para cada duas de corrida e uma de natação. $\mathrm{O}$ baixo volume relativo aos treinos aquáticos deve-se a reduzida atividade da modalidade em relação às demais na competição (FERRACINI, 2015). No entanto, a natação exige maior especificidade mecânica de todas as modalidades, o que promove por vezes algumas lesões (ANJOS, FERNANDES FILHO, NOVAES, 2003). A prova de ciclismo é a mais longa no triathlon, por isso é essencial que o triatleta tenha desempenho similar aos ciclistas de elite (DIEFENTHAELER et al., 2007). Em relação ao tempo efetuado em cada prova, um estudo traz a mensuração de que em três quartos da prova realiza-se a natação e o ciclismo para então iniciar-se a corrida (COSTA; KOKUBUN, 1995). Este total faz com que os desgastes físicos prevaleçam em sua maioria durante as corridas (relatado por $84,2 \%$ dos entrevistados), pois é a fase em que o atleta se encontra sobrecarregado das duas provas anteriores (ONGARATTO; TOIGO, 2010). Além do acúmulo em treinos combinados, a prática da corrida mesmo isolada é apontada pela maioria dos triatletas como a mais desgastante (ZWINGENBERGER et al., 2013). A modalidade exige ampla coordenação biomecânica vinculada ao desempenho de velocidade com choques constantes do corpo contra gravidade (SILVA, FRAGA, GONÇALVES, 2007).

A orientação de treinamento personalizado principalmente realizada por profissionais de Educação Física, destaca-se também em outros estudos que apontam a necessidade do treinamento ser planejado individualmente por profissionais especializados na área em virtude das particularidades e valências de cada atleta (EGERMANN et al., 2003; STROCK; COTTRELL; LOHMAN, 2006). Por sua vez, as avaliações - mais concentradas em cuidados médicos e medições corporais, explicitam as preferências individuais que, aliadas aos principais motivos elencados a prática - acabam por deixar de lado as análises de valências e desempenho (CARNAVAL, 1997). No que se refere às atividades pré e pós sessão, um estudo traz que costuma-se variar entre os indivíduo a opção pela realização de aquecimento e/ou alongamentos, sem impactos diretos no desempenho dos treinos (EGERMANN et al., 2003). Por outro lado, uma pesquisa apresenta que o aquecimento pré-treinamento, praticado pela maioria avaliado neste estudo, pode reduzir o índice de lesões em treinos combinados e competições (BURNS; HUNS; KEENAM, 2003).

$\mathrm{O}$ alto índice de lesões assemelha-se a estudos que encontraram variações de $68,5 \%$ a $75 \%$ em triatletas amadores e elucidam que o principal motivo para tais resultados é o extenso tempo total de prática (PERRONI et al., 2006; WILK; FISHER; RANGELLI, 1995). Um estudo que investigava a ocorrência de lesão por tempo de treino constatou via questionário que dos 
174 atletas participantes, 56\% relataram lesionar-se por excesso de repetições e encontrou que em geral a média de lesões sofridas era de aproximadamente uma a cada mil horas de treino (ANDERSEN et al., 2013). Em outro artigo encontrou-se uma relação entre o total de horas semanais e a ocorrência das lesões, destacando que aqueles que treinavam mais de 15 horas, dobravam seus riscos comparados aos que efetuavam de 11 a 14 horas (SHAW et al., 2004). Além dos malefícios dos rígidos volumes de treinos, como o triathlon é um esporte com três distintas modalidades, seu risco de lesões é ampliado. Um estudo percebeu em uma análise com 152 atletas que possuíam experiência em treinamentos esportivos em uma das três modalidades que compõe o esporte, que antes de praticarem o triathlon $53,4 \%$ não haviam se lesionado em suas atividades prévias ao esporte (BEZEN; BEZEN, 2009).

Assim como esta, pesquisas apresentam em totalidade a predominância das lesões ocorridas durante os treinos em relação à competição (KORKIA; TUNSTALL-PEDOE DPHIL; MAFFULLI, 1994; BURNS; HUNS; KEENAM, 2003; EGERMANN et al., 2003). Averiguando isto, um estudo comparou as características do treinamento em períodos competitivos e não competitivos, e atestou que apesar do baixo índice lesivo durante as competições, os casos tendem a concentrar-se $(78 \%)$ nos treinos do período competitivo, onde neste, mesmo havendo uma média menor de horas semanais totais, os comuns treinos nesse período que envolvem práticas das modalidades combinadas colaborando para o aumento das sobrecargas lesivas (BURNS; HUNS; KEENAM, 2003). Além disso, uma pesquisa verificou que seus 656 triatletas avaliados participavam de seis competições em média por ano, despendendo aproximadamente 30 horas de atividade, enquanto se efetuava perto de 816 horas de reino anuais. Isto ocasiona a proporção de seis vezes mais lesões por tempo de prática competitiva do que em treinamentos, o que além da prática combinada das modalidades agrega o foco constante no desempenho máximo de velocidade (EGERMANN et al., 2003).

Quanto à prevalência de lesões por modalidade, os casos concentramse abrasivamente durante as corridas. Como já mencionado, após todo o trabalho efetuado durante a natação e o ciclismo, é durante a corrida que o corpo mais se esforça, além de ser das três a prática que mais envolve impacto e ajustes corporais constantes durante a movimentação (ANDERSEN et al., 2013; MIGLIORINI, 2011; VLECK et al., 2010). Com isto, a modalidade é a principal responsável pela predominância de lesões nos membros inferiores que ocorrem principalmente nos ossos do pé, tendão do calcâneo, panturrilha, parte posterior da coxa e joelhos (IVKOVIC et al., 2007; SHAW et al., 2004; BERTOLA et al., 2014). Diferentemente deste, a maior parte dos estudos encontrados trazem o joelho como principal alvo de lesões, relatado com motivos relacionados ao uso excessivo da articulação, ainda com a adição das práticas de ciclismo que costumam apresentar incidência lesiva muito inferior às corridas, mas que também contribuem a sobrecarregar os joelhos (SHAW et al., 2004; PERRONI 
et al., 2006; ZWINGENBERGER et al., 2013). A natação é das três a menos lesiva, pois se desenvolve com o menor nível de impacto por suceder-se em meio aquático, contudo, é responsável pela parcela considerável de lesões no manguito rotador (BUSSO, 2008).

As lesões em geral acabam por restringir o uso do local afetado, porém não necessariamente impedem o atleta de prosseguir seus treinos, pois um estudo denota que é comum em casos de lesões nos ombros, por exemplo, que o indivíduo evite a natação, mas que prossiga com as corridas (VLECK et al., 2010). Ademais, além de substituir o exercício, um estudo recomenda que o retorno às atividades seja gradual, prevenindo o atleta de reincidências de lesões. O tempo de recuperação de um local lesionado está relacionado com o cumprimento do tratamento adequado, principalmente no que tange ao repouso, podendo agravar a contusão, interromper o progresso de desempenho e até mesmo vir a afastar o atleta permanentemente do esporte (COSCA; NAVAZIO, 2007; FERRACINI, 2015). Os resultados trouxeram que a maioria dos avaliados se afasta das atividades por mais de cinco semanas, tempo suficiente para inviabilizar todo o processo de treino competitivo.

\section{CONCLUSÃO}

Os resultados obtidos nesta pesquisa trazem um alto percentual de lesão encontrado entre os atletas avaliados. Nas três modalidades praticadas, a corrida se sobressai como a maior causadora de desgastes e ferimentos desencadeados, sobretudo, nos membros inferiores. Com isto, o acompanhamento profissional qualificado se mostra indispensável fazendo com que a carga nos treinos de corrida sejam moderadas dando uma maior importância a desconfortos e possíveis lesões nos membros inferiores.

\section{REFERÊNCIAS}

AGUIAR, P. R. C.; BASTOS, F. N.; JÚNIOR, J. N.; VANDERLEI, L. C. M.; PASTRE, C. M. Lesões Desportivas no Esporte. Revista Brasileira de Medicina do Esporte, v. 16, n. 4, 2010.

ANDERSEN, C. A.; CLARSEN, B.; JOHANSEN, T. V.; ENGEBRETSEN, L. High prevalence of overuse injury among Iron-Distance triathletes. Br J Sports Med, n. 47, p. 857- 861, 2013.

ANJOS, M. A. B.; FERNANDES FILHO, J.; NOVAES, J.S. Características somatotípicas, dermatoglíficas e fisiológicas do atleta de triatlo. Fitness \& Performance Journal, Rio de Janeiro, v.2, n.1, 49-57, 2003.

BATLOUNI, M. Álcool e sistema cardiovascular. Arquivos Médicos do ABC, v. 31, 2006. 
BERTOLA, I. P.; SARTORI, R. P.; CORREA, D. G.; ZATZ, T. G. G.; GOMES, A. R. S. Profile of Injuries Prevalence in Athletes Who Participated in Sesc Triathlon Caiobá. Acta Ortop. Bras., v. 22, n. 4, p.191-196, 2014.

BEZEM, L. S.; BEZEM, S. S. Lesões em triatletas de Ironmen. Revista Brasileira de Prescrição e Fisiologia do Exercício, São Paulo, v.3, n.14, p.110-117, 2009.

BURNS, J.; HONS, B.; KEENAN, A. M.; REDMOND, A. C. Factors Associated With Triathlon-Related Overuse Injuries. Journal of Orthopaedic \& Sports Physical Therapy, v. 33, n. 4, 2003.

BUSSO, G. L. Proposta preventiva para laceração no manguito rotador de nadadores. Revista brasileira de ciência e movimento, v. 12, n. 3, p. 39-46, 2008.

CARNAVAL, P. E. Medidas e avaliação em ciências do esporte. São Paulo: Sprint, 1997.

CBTri, Confederação Brasileira de Triathlon. Site oficial. 2019. Disponível em: <http:/ / www.cbtri.org.br/triathlon/>. Acesso em: 22 jun. 2019.

CIPRIANI, D. J.; SWARTZ, J. D; HODGSON, C. M. Triathlon and the multisport athlete. The Journal of Orthopaedic and Sports Physical Therapy, vol. 27, n. 1, 1998.

COSCA, D.D.; NAVAZIO, F. Common Problems in Endurance Athletes. University of California Davis Sports Medicine Program, Sacramento, California. Americam Family Physician, n. 76, p. 237-44, 2007.

COSTA, J. M. P.; KOKUBUN, E. Lactato sangüíneo em provas combinadas e isoladas do triatlo: possíveis implicações para o desempenho. Revista Paulista de Educação Física, v. 9, n. 2, p. 125-130, 1995.

DIEFENTHAELER, F., CANDOTTI, C. T., RIBEIRO, J., OLIVEIRA, A. R. Comparação de respostas fisiológicas absolutas e relativas entre ciclistas e triatletas. Revista Brasileira de Medicina do Esporte, v. 13, n. 3, p. 205-208, 2007.

EGERMANN, M.; BRACARI, D.; LILL, C. A.; SCHMIDT, H. Analysis of Injuries in Long Distance Triathletes. International Journal of Sports Medicine, v. 24, p. 271-276, 2003.

EPIPHANIO, E. H.; AMATUZZI, M. M. A opção pelo triatlo como uma prioridade de vida: um estudo fenomenológico. Estudos de Psicologia, v. 14, n. 1, p. 61-70, 1997.

ETXEBARRIA, N.; MUJIKA, I.; PYNE, D. B. Training and Competition Readiness in Triathlon. Sports, v. 7, n. 5, p. 101, 2019.

FERRACINI, S. H. F. Prevalência de lesões em triathlon de longa distância Ironman ${ }^{\circledR}$. 2015. Dissertação (Mestrado em Educação Física) - Universidade Estadual de Campinas, Campinas, São Paulo, 2015. 
GUIMARÃES, V. V. et al. Consumo abusivo e dependência de álcool em população adulta no Estado de São Paulo, Brasil. Revista Brasileira de Epidemiologia, v. 13, p. 314-325, 2010.

ISHIDA, J; de C. et al. Presença de fatores de risco de doenças cardiovasculares e de lesões em praticantes de corrida de rua. Revista brasileira de educação física e esporte, v. 27, n. 1, p. 55-65, 2013.

IVKOVIC', A., FRANIC', M., BOJANIC', I., PECINA, M. Overuse Injuries in female Athletes. Croatian Medical Jounal, n. 48, p. 767-78, 2007.

JACOMEL, G. F.; FREITAS, E. dos S.; RUSCHEL, C.; SCHÜTZ, G. R.; ARAÚJO, L. G. Incidência de lesões em triatletas de longa distância. Coleção Pesquisa em Educação Física, vol.7, n. 3, 2008.

JUNIOR, M. M. da S.; MILARES, L. P. G. R. Lesões no triathlon: uma revisão de literatura. EFDeportes.com: Revista Digital. Buenos Aires, ano 21, n. 215, 2016.

KORKIA P.K, TUNSTALL-PEDOE DPHIL D.S, MAFFULLI N. An epidemiological investigation of training and injury patterns in British triathletes. British Journal of Sports Medicine, v. 28, n.3, p. 191-196, 1994.

MCHARDY, A.; POLLARD, H; FERNANDEZ, M. Triathlon injuries: A review of the literature and discussion of potential injury mechanisms. Clinical Chiropractic, vol. 9, n. 3, 2006.

MELONI, J. N.; LARANJEIRA, R. Custo social e de saúde do consumo do álcool. Revista brasileira de psiquiatria, v. 26, p. 7-10, 2004.

MIGLIORINI, Sergio. Risk factors and injury mechanism in Triathlon. Journal of Human Sport and Exercise, v. 6, n. 2, p. 309-314, 2011.

NAZARIO, P. F. et al. Motivos de prática do triatlo: um estudo com atletas de Santa Catarina. Caderno de Educação Física e Esporte, v. 10, n. 19, p. 55-60, 2011.

ONGARATTO, D.; TOIGO, A. M. Prevalência de lesões musculoesqueléticas em atletas amadores de triatlo federados no Estado do Rio Grande do Sul, Brasil. EFDeportes.com: Revista Digital. Buenos Aires, ano 15, n. 150, 2010.

PERRONI, M. G.; BARONI, B. M.; OLTRAMARI, J. D.; STEDILE, N. R. de A.; LEAL JÚNIOR, E. P.; VANIN, A. A.; DE GODOI, V. Estudos de Lesões em Atletas de Triathlon. Revista Brasileira de Medicina do Esporte, v. 12, n. 6, 2006.

PORTAL, M. N. D., FONSECA, C. L. T., DE OLIVEIRA, A. L. B., DA SILVA SEQUEIROS, J. L., DE OLIVEIRA, E. F., DE ARÊDES, S. G., ... \& DANTAS, E. H. M. Predominância do tipo de fibra muscular e sua relação com a capacidade aeróbica de corredores de provas de fundo. Fitness \& Performance journal, n. 4, p. 211-217, 2004.

RABAHI, M. F. Tuberculose e tabagismo. Pulmão RJ, v. 21, n. 1, p. 46-49, 2012. 
RAMA, J. D. L. S. da. Planeamento do treino no triatlo: um estudo de caso. 2011. Tese de Doutorado. 2011.

SHAW, T.; HOWAT, P.; TRAINOR, M., MAYCOCK. Training Patterns and Injuries in Triathletes. J. Sci. Med. Sport. v. 7, n. 4, p. 446-450, 2004.

SILVA, Sarah Regina Dias; FRAGA, Carina Helena Wasem; GONÇALVES, Mauro. Efeito da fadiga muscular na biomecânica da corrida: uma revisão. Motriz. Journal of Physical Education. UNESP, p. 225-235, 2007.

STROCK, G. A.; COTTRELL, E. R.; LOHMAN, J. M. Triathlon. Physical Medicine and Rehabilitation Clinics of North America, n. 17, p. 553-564, 2006.

VECHIATO, Tauane; DA COSTA, Tatienne Neder Figueira. Avaliação do estado de hidratação e ingestão hídrica em praticantes de triatlo. Revista Brasileira de Nutrição Esportiva, v. 10, n. 57, p. 250-259, 2016.

VLECK, V. E.; BENTLEY, D. J.; MILLET, G. P., COCHRANE, T. Triathlon Event Distance Specialization: Training and Injury Effects. Journal of Strength and Conditioning Research v. 24, n. 1, p. 30-36, 2010.

WELLMANN, Jürgen et al. Changes in alcohol intake and risk of coronary heart disease and all-cause mortality in the MONICA/KORA-Augsburg cohort 1987-97. European Journal of Cardiovascular Prevention \& Rehabilitation, v. 11, n. 1, p. 48$55,2004$.

WILK, B.R.; FISHER, K.L; RANGELLI, D. The Incidence of Musculoskeletal Injuries in an Amateur Triathlete Racing Club. Journal of Orthopedic \& Sports Physical Therapy, v. 22, n. 3, 1995.

YUSUF, Salim et al. Effect of potentially modifiable risk factors associated with myocardial infarction in 52 countries (the INTERHEART study): case-control study. The lancet, v. 364, n. 9438, p. 937-952, 2004.

ZWINGENBERGER, S.; VALLADARES, R. D.; WALTHER, A.; BECK, H.; STIEHLER, M.; KIRSCHNER, S.; ENGELHARDT, M.; KARSTEN, P. An epidemiological investigation of training and injury patterns in triathletes. Journal of Sports Sciences, v. 32, n. 6, p. 583- 590, 2013. 